\title{
Ações voltadas para a atenção farmacêutica hospitalar em um programa de residência multiprofissional no interior da Amazônia, Santarém, Pará
}

\author{
Actions aimed at hospital pharmaceutical care in a multidisciplinary residency program in the \\ interior of the Amazon, Santarém, Pará \\ Acciones dirigidas a la atención farmacéutica hospitalaria en un programa de residencia \\ multiprofesional en el interior de la Amazonía, Santarém, Pará
}

\author{
Ana Elizabete Xavier Fonseca \\ ORCID: https://orcid.org/0000-0002-4448-871X \\ Universidade do Estado do Pará, Brasil \\ E-mail: anaelizastm@gmail.com \\ Daliane Ferreira Marinho \\ ORCID: https://orcid.org/0000-0003-3849-1215 \\ Universidade do Estado do Pará, Brasil \\ E-mail: daliane.marinho@uepa.br \\ Sheyla Mara Silva de Oliveira \\ ORCID: https://orcid.org/0000-0001-6666-2363 \\ Universidade do Estado do Pará, Brasil \\ E-mail: sheylaoliveira@uepa.br \\ Teógenes Luiz Silva da Costa \\ ORCID: https://orcid.org/0000-0001-7040-7939 \\ Universidade Federal do Oeste do Pará, Brasil \\ E-mail: teogenes.costa@ufopa.edu.br \\ Marina Smidt Celere Meschede \\ ORCID: https://orcid.org/0000-0002-6519-9466 \\ Universidade Federal do Oeste do Pará, Brasil \\ E-mail: marcelere@yahoo.com.br \\ Nádia Vicência do Nascimento Martins \\ ORCID: https://orcid.org/0000-0002-8166-644X \\ Universidade do Estado do Pará, Brasil \\ E-mail: nadia.martins@uepa.br \\ Lívia de Aguiar Valentim \\ ORCID: https://orcid.org/0000-0003-4255-8988 \\ Universidade do Estado do Pará, Brasil \\ E-mail: livia.valentim@uepa.br
}

\begin{abstract}
Resumo
As residências multiprofissionais em saúde são programas de qualificação profissional que permitem a integração do discente com profissionais de diferentes áreas por meio da atuação em campos de práticas, estimulando o pensamento reflexivo, crítico, humanístico e holístico. Em residências multiprofissionais o farmacêutico poderá ser um dos integrantes que compõe a equipe e deverá participar na gestão, farmacotécnica medicamentosa e na clínica, como em ambientes críticos e de alta complexidade destacando-se a prática essencial na atenção farmacêutica hospitalar. Diante do apresentado, este artigo visa relatar as experiências de um residente farmacêutico em um programa multiprofissional voltado para a atenção integral em Ortopedia e Traumatologia, realizado em um Hospital Regional na região do Baixo Amazonas, cidade de Santarém. Durante a experiência da residência o farmacêutico pode executar a clínica farmacêutica assim como a gestão e lidando com as diversas situações cotidiano de um hospital, tendo a oportunidade de acompanhar e construir um plano terapêutico que acompanha a evolução do paciente durante toda a sua estadia no hospital. Observase que o residente muitas vezes é exposto a diferentes casos, tendo suporte e apoio para superar suas inseguranças, tendo um contato direto e decisivo no tratamento clínico do paciente, sendo um membro atuante da equipe hospitalar, encontrando na residência um ambiente fértil para o aprendizado e especialização.
\end{abstract}

Palavras-chave: Assistência farmacêutica; Serviço de farmácia hospitalar; Relações profissional-paciente.

\section{Abstract}

Multiprofessional residencies in health are professional qualification programs that allow the integration of students with professionals from different areas by working in fields of practice, stimulating reflective, critical, humanistic and holistic thinking. In multi-professional residencies, the pharmacist may be one of the members that make up the team 
and must participate in management, drug and clinical pharmacotechnics, as well as in critical and highly complex environments, highlighting the essential practice in hospital pharmaceutical care. Given the above, this article aims to report the experiences of a pharmacist resident in a multidisciplinary program focused on comprehensive care in Orthopedics and Traumatology, held at a Regional Hospital in the Lower Amazon region, city of Santarém. During the residency experience, the pharmacist can perform the pharmaceutical clinic as well as manage and deal with the various daily situations of a hospital, having the opportunity to monitor and build a therapeutic plan that follows the patient's evolution throughout their stay in the hospital. It is observed that the resident is often exposed to different cases, having support and support to overcome their insecurities, having a direct and decisive contact in the clinical treatment of the patient, being an active member of the hospital team, finding a fertile environment in the residence. learning and specialization.

Keywords: Pharmaceutical services; Pharmacy service hospital; Professional- patient relations.

\section{Resumen}

Las residencias multiprofesionales en salud son programas de cualificación profesional que permiten la integración de estudiantes con profesionales de diferentes áreas trabajando en campos de práctica, estimulando el pensamiento reflexivo, crítico, humanístico y holístico. En residencias multiprofesionales, el farmacéutico puede ser uno de los miembros que componen el equipo y debe participar en la gestión, farmacotecnia farmacéutica y clínica, así como en entornos críticos y de alta complejidad, destacando la práctica imprescindible en la atención farmacéutica hospitalaria. Dado lo anterior, este artículo tiene como objetivo relatar las experiencias de un farmacéutico residente en un programa multidisciplinario enfocado a la atención integral en Ortopedia y Traumatología, realizado en un Hospital Regional de la región de la Baja Amazonía, ciudad de Santarém. Durante la experiencia de la residencia, el farmacéutico puede realizar la clínica farmacéutica así como gestionar y afrontar las distintas situaciones cotidianas de un hospital, teniendo la oportunidad de monitorizar y construir un plan terapéutico que sigue la evolución del paciente a lo largo de su estancia en el hospital. Se observa que el residente suele estar expuesto a diferentes casos, teniendo apoyo y apoyo para superar sus inseguridades, teniendo un contacto directo y decisivo en el tratamiento clínico del paciente, siendo miembro activo del equipo hospitalario, encontrando en la residencia un ambiente fértil para el aprendizaje y la especialización.

Palabras clave: Cuidado farmacéutico; Servicio de farmacia hospitalaria; Relaciones profesional-paciente.

\section{Introdução}

O farmacêutico é um profissional que integra as categorias de programas de residências multiprofissionais em saúde no Brasil e poderá atuar em diferentes níveis de atenção do Sistema Único de Saúde (SUS), dentre eles o terciário, desenvolvendo ações voltadas para a farmácia hospitalar, a farmacovigilância, o controle de estoque e no sistema de garantia de qualidade permeando a atenção farmacêutica (Messeder et al, 2007).

A atenção farmacêutica é considerada como uma nova prática profissional, vinculada de forma exclusiva ao farmacêutico, traduzida na responsabilidade e no compromisso com a resolução de problemas farmacológicos complexos (Agonesi \& Sevalho, 2010). Na atenção farmacêutica o paciente é colocado ao centro do cuidado e o profissional tem o papel de aconselhar, monitorar a terapia farmacológica, garantindo a adesão do tratamento e o uso racional do medicamento, podendo detectar possíveis suspeitas de problemas relacionados a medicamentos, a fim de buscar maneiras de amenizar reações adversas e uma terapia segura (Silva, 2018).

Segundo Provin e colaboradores (2009), na atenção farmacêutica ocorre uma mudança de ênfase/foco do produto (medicamento) para o indivíduo e muitos profissionais ainda não apresentam consciência clara de suas funções. Dessa forma, torna-se importante à formação e a capacitação do profissional farmacêutico são pontos importantes e que devem apresentar uma concepção crítica e diferenciada para que possa de fato exercer a atenção farmacêutica em seu local de trabalho (Agonesi \& Sevalho, 2010).

No contexto da atenção farmacêutica hospitalar, o farmacêutico é o responsável pela terapêutica medicamentosa hospitalar, apresenta uma posição ativa com a possibilidade de contribuir com melhorias em relação ao tratamento do paciente, como por exemplo, por meio de práticas clínicas, participando de comissões para controle de infecção hospitalar (CCIH) e redução de custos desnecessários, bem como, do uso racional de medicamento (Felipe et al, 2018). A inserção do profissional farmacêutico dentro do contexto hospitalar demanda orientação, comunicação e discussão com a equipe multiprofissional, o farmacêutico atua como um facilitador da aprendizagem, tendo a função de informar sobre o uso do medicamento; criando as 
condições para que o paciente às adquira de forma proveitosa e efetiva, organizando estratégias para o aprendizado da terapia prescrita (Possamai \& Dacoreggio, 2007).

Em uma perspectiva multidisciplinar, a assistência farmacêutica no âmbito hospitalar, engloba para além da atuação do profissional farmacêutico, mas também, outros profissionais do campo da saúde como médicos, enfermeiros, fisioterapeutas, bem como, as tecnologias e os saberes das ciências sociais (Osorio-de-Castro et al, 2017). Ao longo do tempo, o campo da assistência farmacêutica vem se aprimorando e agrega novos saberes, conhecimentos, conformando-se como um campo multiprofissional e multissetorial que evidencia interfaces (Osorio-de-Castro et al, 2017), sendo ampliada a oferta de vagas dentro dos programas de residências multiprofissionais, por entender a importância desse profissional, seja no ambiente hospitalar, os nos serviços de prevenção e promoção de saúde. O cuidado multiprofissional em saúde tem sido apontado como uma estratégia para enfrentar o intenso processo de especialização na área da saúde (Peduzzi, 2001).

Nessa perspectiva de integrar uma equipe multidisciplinar no âmbito hospitalar, o farmacêutico contribui com outros profissionais na assistência preventiva, tomando decisões e atuando na diminuição da incidência de erros no processo dinâmico e multidisciplinar, na análise da prevalência de erros na administração de medicamentos em ambiente hospitalar, promovendo saúde monitorando possíveis eventos adversos. (Borges, 2019)

Os programas de residência multiprofissionais em saúde desenvolvidos no contexto hospitalar, possibilitam que o farmacêutico, em geral um profissional recém-formado, participe e se qualifique de forma crítica e reflexiva nesse cenário de atuação de forma bastante positiva. Segundo Silva (2018) os programas de residência multiprofissionais enaltecem uma possibilidade de formação em saúde atenta e associada à diversidade e complexidade das necessidades de saúde postas no cotidiano do SUS. As residências multiprofissionais em saúde voltadas para a área hospitalar, possibilitam ao farmacêutico, vivenciar as ações da atenção e assistência farmacêutica de durante os dois anos de sua formação, qualificando-o para o mercado de trabalho. Entretanto, na literatura pouco é divulgado sobre essas atividades, a escassez de informações sobre o papel do farmacêutico em programas de residência hospitalares limita o aperfeiçoamento dos programas e a construção aprimorada da formação Lato sensu em residência.

Diante do apresentado, este trabalho tem como objetivo relatar as principais experiências vivenciadas por farmacêuticos residentes inseridos no contexto da farmácia hospitalar em um programa residência multiprofissional em saúde localizado no interior da Amazônia, Santarém, estado do Pará no ano de 2021.

\section{Metodologia}

Este artigo é resultado de uma investigação do tipo qualitativa, uma pesquisa de campo (Gil, 1999), escrito no formato de relato de experiência onde se buscou descrever as principais vivências do residente farmacêutico inserido no campo de prática da farmácia hospitalar em um programa multiprofissional, na cidade de Santarém, durante o primeiro semestre do ano de 2021. A ferramenta metodológica utilizada foi a observação participante mediada, do ponto de vista teórico, pelo conceito de descrição densa (Geertz, 1989).

Para Geertz, a pesquisa social deveria apresentar uma descrição acurada da realidade social, recorrendo ao exemplo de Gilbert Ryle, com o famoso caso hipotético da piscadela realizada por duas pessoas distintas, em que temos a seguinte situação, "um [sofre de um] tique involuntário; no outro, é uma piscadela conspiratória a um amigo" (Geertz, 1989). Se os dois indivíduos, piscando ao mesmo tempo, fossem observadas em laboratório, descolados de sua realidade, ninguém poderia dizer qual delas seria um tique nervoso ou uma piscadela ou, na verdade, se ambas eram piscadelas ou tiques nervosos (Geertz, 1989). Seria fundamental observar os sujeitos em seus contextos sociais, em seu cotidiano, na teia de relações à qual estão vinculados para compreender qual dos indivíduos pisca involuntariamente em razão do tique nervoso, e qual deles é usa a piscadela de maneira conspiratória. 
Segundo Geertz, a cultura, no que se refere à sua dimensão objetiva, pode ser considera um texto, um dado que pode ser observado. Enquanto texto os comportamentos culturais podem ser, assim como textos escritos, interpretados. Dessa maneira, os investigadores devem, mediante a observação profunda da realidade social, produzir uma descrição densa que subsidie a interpretação da cultura em observação. Assim, nesse texto apresentamos uma interpretação dos acontecimentos observados durante as atividades de residência.

A farmácia hospitalar, local utilizado para relatar as vivências, está localizada em um Hospital Público de cunho regional, considerado o de maior porte na cidade de Santarém, responsável por atender populações da região do Baixo Tapajós, no estado do Pará. A região do Baixo Tapajós, segundo dados do Governo do estado do Pará, é compreendida pelos municípios de Alenquer, Almeirim, Belterra, Terra Santa, Oriximiná, Óbidos, Prainha, Curuá, Mojuí dos Campos, Monte Alegre, Faro, Santarém e Juruti (SESPA, 2016).

\section{Resultados e Discussão}

\subsection{O relato de experiência do ambiente de trabalho de farmácia hospitalar}

O primeiro momento da experiência da Residência é a realização do acolhimento, treinamentos e inserção dos residentes na rotina do hospital e as funções do farmacêutico. Cada residente é dimensionado para um setor onde o farmacêutico da equipe irá realiza a função de preceptor, treinando para the oferecer o suporte nas atividades exercidas, é importante destacar que alguns farmacêuticos receberam o curso de preceptoria e que essa é uma prática comum dentro da rotina do hospital de ter algum profissional mais experiente para acompanhar um novo colaborador o que torna o processo de adaptação, mas produtivo.

Muitos residentes são recém-formados e não se sentem totalmente seguros para lidar com a prática a que vão ser expostos, o que ocasiona tanto inquietações e inseguranças como o amadurecimento. Sendo importante o acolhimento para que estes se familiarizem e se identifiquem com o seu novo ambiente. A prática da residência possibilita a construção de conhecimentos com base em trabalho em equipe, planejamento de ações, relações interpessoais, ações dialéticas, troca de saberes, renovação de atitudes, autoanálise, escuta, sendo este um processo intenso, dinâmico e desbravador dentro de um campo de atuação enriquecedor, fértil e estimulante (Lucas et al., 2016).

A farmácia clínica realizada neste Hospital regional é bastante atuante e acompanha o paciente desde a sua entrada com o processo de admissão farmacêutica, passando por toda a sua estadia dentro do hospital, traçando um plano de cuidado até o momento de sua alta hospitalar em que o paciente necessita realizar a alta farmacêtica.

Durante o processo de admissão o farmacêutico residente tem a possibilidade de conversar e ter o contato direto com o paciente, criando a habilidade clínica nesse período. O residente vai até o paciente ou o seu acompanhante recolher informações sobre o seu estado clínico, seu diagnóstico, ouvido suas queixas e o relato do próprio paciente sobre sua situação, este momento costuma ser desafiador é também importante para a construção de um plano terapêutico voltado para cada paciente, tendo ele como foco principal, é importante tanto para o paciente quanto para a equipe assistencial colaborando para uma terapia farmacológica eficiente e segura usando as habilidades e percepções do farmacêutico nesse momento.

Durante a anamnese farmacêutica, o farmacêutico pode contribuir para a terapêutica do paciente dentro do hospital colhendo informações sobre a sua história farmacoterapêutica como por exemplo, se este paciente possui alguma alergia medicamentosa, se possui algum medicamento de uso contínuo, se possui alguma doença preexistente, ou se há alguma queixa durante sua estadia. Nesse momento o farmacêutico residente exercita a sua habilidade clínica percebendo em cada caso em que pode intervir para evitar problemas relacionados ao medicamento ou contribuir para uma terapêutica mais eficaz. A partir desse momento o residente tem a possibilidade de acompanhar a evolução desse paciente através de visitas clínicas definidas a partir de um plano terapêutico.

O acompanhamento da evolução clínica do paciente através das visitas contribui não só pela melhora clínica do paciente, 
dando a possibilidade de o farmacêutico perceber e evitar problema na farmacoterapia do paciente criando um vínculo de confiança com a equipe que percebe a importância do farmacêutico e com o paciente que observa a preocupação da equipe com o seu bem estar. Além das visitas a cada dia é verificada a prescrição diária do paciente, sendo este também um momento em que o farmacêutico avalia a melhor forma de ser realizado o tratamento farmacológico de cada paciente.

$\mathrm{Na}$ avaliação das prescrições médicas diárias, o farmacêutico pode evitar interações medicamentosas, através de aprazamentos corretos dos medicamentos, ajustar doses de acordo com o estado clínico do paciente, aconselhar inclusão ou a exclusão de medicamentos que possam contribuir com a melhor estratégia terapêutica voltada para a situação particular do paciente. Esse momento de integração tanto com a equipe de enfermagem que fica responsável de administrar os medicamentos quanto com o médico prescritor, sendo que as sugestões de intervenções farmacêuticas são discutidas e esclarecidas entre estes membros. É possível perceber a importância dessas intervenções, sendo que estas são aceitas e reconhecidas como importantes na recuperação do paciente durante sua estadia no hospital até o momento de sua alta, que também é acompanhada pelo farmacêutico.

O momento da alta hospitalar o farmacêutico é um dos componentes que auxiliam esse momento, atuando no momento posterior a estadia hospitalar, quando é importante que o paciente continue tomando medidas de cuidado para a sua plena recuperação, é nesse momento em que o farmacêutico dá as orientações ao paciente e ao seu acompanhante sobre o uso adequado das medicações de prescrições de alta hospitalar, tendo em vista o uso racional do medicamento e o autocuidado do paciente. É um momento desafiador e recompensador, para o residente que acompanha toda a estadia do paciente e é mais um momento de exercício da farmácia clínica e de sua contribuição com a equipe multiprofissional.

O farmacêutico clínico tem adquirido mais espaço na equipe multidisciplinar devido sobretudo aos valores das intervenções ocasionadas por sua presença qualificada e atuante. As intervenções farmacêuticas estão cada vez mais aceitas no contexto terapêutico e evidencia importância do profissional farmacêutico clínico na assistência direta ao paciente, assim como sua contribuição na promoção de resultados clínicos mais satisfatórios, mediante rotinas sistemáticas de avaliação farmacêutica da prescrição médica e o estímulo à prescrição segura e racional. (Dos Santos et al., 2020)

A evolução da atuação do farmacêutico no cuidado intensivo por exemplo, vem passando da logística e dispensação de medicamentos (ações reativas) para a participação clínica efetiva junto à equipe multiprofissional (ações proativas), como pro exemplo o ajuste de dose, a suspensão da terapia e a recomendação de início de terapia resultando numa maior valorização das recomendações farmacêuticas na prática clínica. Sendo possível pela evolução do conhecimento clínico do farmacêutico e por uma maior integração à equipe multiprofissional da UTI. A participação mais ativa do farmacêutico na terapia farmacológica do paciente grave vem sendo uma prática desejável e deve envolver não somente a provisão de assessoria terapêutica para a equipe, como também a participação ativa nas decisões sobre manutenção da farmacoterapia. (Fideles et al., 2015)

\subsection{O relato referente a gestão de medicamentos na farmácia hospitalar e os desafios impostas durante a pandemia}

Um dos grandes desafios enfrentados pelos residentes e compartilhado por toda a equipe assistencial é a prestação do serviço de saúde durante a pandemia do vírus COVID-19 nos anos de 2020 e 2021. A pandemia modificou todos os processos internos dos hospitais, foram feitas adaptações para assegurar a segurança, qualidade e eficácia dos tratamentos dos pacientes atendidos mesmo com a gravidade a que o contexto da pandemia nos impõem.

Certamente o aumento de pacientes e muitos deles em estado grave é um dos desafios mais significativos. Para a farmácia a gestão de medicamentos é essencial para evitar o desabastecimento, para tanto foi adotado algumas medidas como o controle de medicamentos, principalmente sedativos, a qual foram confeccionados de kits de sedação, e avaliado o número diária de pacientes sedados por setor, assim contribuindo para o controle de estoque.

Na parte da farmácia clínica o desafio foi realizar a farmácia assistencial, garantindo a segurança tanto do paciente quanto do profissional, muitos dos pacientes isolados não se é permitido as visitas e para não se expor o farmacêutico teve que 
reduzir a frequência de visitas, realizar a evolução do paciente por meio do prontuário eletrônico do paciente e coletar informações pertinentes com a equipe médica ou de enfermagem. Nesse contexto a comunicação com a equipe multiprofissional foi essencial

A presença de residentes pode proporcionar um aumento da capacidade da equipe de resolver e/ou enfrentar os problemas cotidianos, pois, para os colaboradores, os residentes podem representar um apoio na prestação do cuidado, por meio da troca de conhecimentos, discussão de casos e tomada de decisão entre diferentes categorias profissionais. A inserção da residência, amplia a relação teoria prática, fortalece ações de saúde de acordo com a integralidade da atenção, se tornando um dispositivo de mudança do modelo de assistência à saúde. Assim como, no decorrer da residência, os residentes são modificados pelas situações vivenciadas e experienciadas no dia a dia, não somente em relação à técnica, mas na habilidade de se relacionar com os profissionais e pacientes. (Domingos et al., 2015)

\subsection{Intervenção farmacêutica realizadas pelo residente e as singularidades do atendimento no contexto da Amazonas}

O processo de integração não só com a equipe de farmácia como também com a equipe assistencial é realizada em conjunto com o preceptor em um primeiro momento, porém quando já há mais habilidade do residente esse processo é realizado por si só, é importante lembrar que a multidisciplinaridade é uma das partes principais das RMPS, mitigando muitas vezes deficiências encontradas na própria formação, no caso do farmacêutico habilidades como o olhar crítico e reflexivo além de humanizado e holístico para o paciente e a integração com a equipe médica e de enfermagem são ações encorajadas durante a práticas da residência.

No contexto da Amazônia essa integração entre a equipe hospitalar é importante para um entendimento integral da situação de cada paciente. O hospital Regional é um centro de referência para diversas cidades do Baixo Amazonas, assim o residente tem a oportunidade de entrar em contato com diferentes casos, de pacientes que estão enfrentando um tratamento de de média e alta complexidade, com aspectos socioculturais diferentes e que muitas vezes estão longe de suas residências e comunidades. Entender as dificuldades e valorizar os determinantes sociais em saúde que acompanham cada paciente é um fator importante para auxiliar na recuperação de cada caso, exercitando um olhar holístico e integral que é uma das práticas incentivadas durante as RMS.

As Residências Multiprofissionais em Saúde constituem uma estratégia de formação de recursos humanos para o Sistema Único de Saúde. A elevada carga horária, a diversidade de cenários e a complexidade das situações vivenciadas na Residência preparam o profissional para os desafios de sua futura atuação, o processo de compartilhar os saberes e a troca das especificidades amplia o olhar de cada profissional e resulta no objetivo comum e central do cuidado integral ao paciente. A busca pela clínica ampliada, a multiprofissionalidade e a interdisciplinaridade vivenciadas no ambiente hospitalar permitem aos residentes reconhecer a importância fundamental da atuação de cada profissão ao juntar os saberes específicos para a melhor condução/intervenção. Além disso, é imprescindível o (re)conhecimento do histórico de vida de cada paciente para melhor intervenção e adesão ao cuidado proposto (Alves et al., 2016).

A formação dos residentes, destaca-se por dialogar sobre a educação permanente e assim contribuir para o desenvolvimento de competências para os profissionais de saúde em diferentes cenários, principalmente o SUS. Estimula-se que os residentes adquiram atitudes críticas reflexivas e atuem como articuladores participativos, criando alternativas estratégicas e inovadoras na atenção e gestão indispensáveis para as mudanças que visam a consolidação do SUS (Silva et al., 2016).

\section{Conclusão}

O farmacêutico inserido na residência passa a ter uma atividade prática no de atenção farmacêutica e hospitalar, com uma visão mais ampla dessas práticas que são repassadas muitas vezes de forma rápida e/ou superficial durante a graduação. A 
prática hospitalar verifica-se a importância do farmacêutico não apenas na farmacotécnica ou gestão dos insumos farmacêuticos como também na farmácia clínica contribuindo com a terapêutica e qualidade de vida do paciente, evitando problemas relacionados ao medicamento, e contribuindo com o uso racional do medicamento atuado de forma decisiva nas condutas clínicas, tendo o medicamento como sua principal ferramentas para o atendimento do paciente.

As ações voltadas para a atenção farmacêutica hospitalar contribuem com efetivo tratamento farmacológico do paciente desde a sua entrada do hospital até o momento de sua alta, o farmacêutico contribui com uma terapêutica mais segura e eficaz estabelecendo um vínculo com o paciente e com toda a equipe assistencial. Esse processo pode ser desafiador e enriquecedor no contexto da residência multiprofissional em saúde contribuindo para o amadurecimento profissional do farmacêutico inserido na RMS.

No contexto da Amazônia é importante levar em conta os determinantes sociais em saúde que acompanham o paciente, muitas vezes, o residente deve exercitar um olhar integral para entender o contexto social e cultural de cada paciente e como isso pode afetar a seu tratamento dentro e fora do hospital. Sendo exercido aqui um dos objetivos das residências multiprofissionais. O olhar crítico, reflexivo e holístico dos profissionais que estão atuando nos serviços de saúde.

$\mathrm{O}$ residente muitas vezes é exposto a diferentes casos, tendo suporte e apoio para superar suas inseguranças, tendo um contato direto e decisivo no tratamento clínico do paciente, proporcionando uma habilidade assistencial essencial para a recuperação da saúde do paciente, sendo um membro atuante da equipe hospitalar, encontrando na residência um ambiente fértil para o aprendizado e especialização assim como contribui de forma significativa com a equipe farmacêutica.

\section{Referências}

Alves, C. C., Netto, M. C., Sousa, A. P. G. D., \& Devincenzi, M. U. (2016). Relato de experiência da atuação do nutricionista em Residência Multiprofissional em Saúde. Revista de Nutrição, 29, 597-608.

Angonesi, D., \& Sevalho, G. (2010). Atenção Farmacêutica: fundamentação conceitual e crítica para um modelo brasileiro. Ciência \& saúde coletiva, 15, 36033614 .

Borges, M. V. (2019). O papel do farmacêutico clínico na atenção farmacêutica hospitalar. Ariquemes: FAEMA. 39p.

Castro, C. G., \& Castilho, S. R. (2004). Diagnóstico da farmácia hospitalar no Brasil. In Diagnóstico da farmácia hospitalar no Brasil (pp. 150-150).

Clifford, G. E. E. R. T. Z. (1989). A interpretação das culturas. Rio de Janeiro: LTC-Livros Técnicos e Científicos Editora.

da Silva, L. C. A., de Brito, P. O. L., Melo, C. D., Falcai, A., \& de Paiva Pereira, I. C. (2018). Contribuições da atenção farmacêutica a pacientes em tratamento oncológico. Revista de Investigação Biomédica, 9(2), 210-217.

Domingos, C. M., Nunes, E. D. F. P. D. A., \& Carvalho, B. G. (2015). Potencialidades da Residência Multiprofissional em Saúde da Família: o olhar do trabalhador de saúde. Interface-Comunicação, Saúde, Educação, 19, 1221-1232.

dos Santos, J. P., dos Santos, R. M. D. H., de Araújo, P. L., Bendicho, M. T., \& Xavier, R. M. F. (2020). Cuidado farmacêutico em UTI oncológica. Brazilian Journal of Health Review, 3(3), 5697-5704.

Felipe, R. L., Guimarães, S. T., Franqueiro, E. P. M., \& Cardoso, R. A. (2018). O Papel Do Farmacêutico Clínico No Âmbito Hospitalar: Uma Revisão Bibliográfica. $e-R A C, 8(1)$.

Fideles, G. M. A., Alcântara-Neto, J. M. D., Peixoto, A. A., Souza-Neto, P. J. D., Tonete, T. L., Silva, J. E. G. D., \& Neri, E. D. R. (2015). Recomendações farmacêuticas em unidade de terapia intensiva: três anos de atividades clínicas. Revista Brasileira de Terapia Intensiva, 27, $149-154$.

Gil, A. C. (1999). Como elaborar projetos de pesquisa. Atlas, 2002. Métodos e técnicas de pesquisa social, 6, 22-23.

Lucas, Á. B., de Freitas, N. A., Freitas, F. D., da Ponte Neto, O. A., Lacerda, R. A., \& Cavalcante, V. O. M. (2016). Sentimentos que transpõem a residência multiprofissional em saúde da família: relato de experiência. SANARE-Revista de Políticas Públicas, 15(2).

Messeder, A. M., Osorio-de-Castro, C. G. S., \& Camacho, L. A. B. (2007). Projeto Diagnóstico da Farmácia Hospitalar no Brasil: uma proposta de hierarquização dos serviços. Cadernos de Saúde Pública, 23, 835-844

Osorio-de-Castro, C. G. S., Oliveira, M. A., \& Vasconcelos, D. M. M. D. (2017). Assistência Farmacêutica: um campo em consolidação.

Peduzzi, M. (2001). Equipe multiprofissional de saúde: conceito e tipologia. Revista de saúde pública, 35, $103-109$.

Provin, M. P., Campos, A. D. P., Nielson, S. E. D. O., \& Amaral, R. G. (2010). Atenção Farmacêutica em Goiânia: inserção do farmacêutico na Estratégia Saúde da Família. Saúde e Sociedade, 19, 717-724. 
Research, Society and Development, v. 10, n. 12, e434101220711, 2021

(CC BY 4.0) | ISSN 2525-3409 | DOI: http://dx.doi.org/10.33448/rsd-v10i12.20711

Possamai, F. P., \& Dacoreggio, M. D. S. (2007). A habilidade de comunicação com o paciente no processo de atenção farmacêutica. Trabalho, educação e saúde, 5, 473-490.

SESPA. Secretaria do Governo do estado do Pará. Polo Tapajós. http://www.setur.pa.gov.br/polo-tapajos.

Silva, C. T. D., Terra, M. G., Kruse, M. H. L., Camponogara, S., \& Xavier, M. D. S. (2016). Residência multiprofissional como espaço intercessor para a educação permanente em saúde. Texto \& Contexto-Enfermagem, 25.

Silva, L. B. (2018). Residência Multiprofissional em Saúde no Brasil: alguns aspectos da trajetória histórica. Revista Katálysis, 21, $200-209$. 